\title{
Pancreatic Ewings Sarcoma- A Dreadful Tumor
}

\author{
Kumar Jayant $^{1, *}$, Swati Agrawal ${ }^{2}$, Rajendra Agarwal $^{3}$, Susheela Khoiwal ${ }^{4}$ \\ ${ }^{1}$ Department of Surgery, Sudha Hospital \& Medical Research Centre, Talwandi, Kota, Rajasthan, India \\ ${ }^{2}$ Sudha Hospital \& Medical Research Centre Kota, Rajasthan, India \\ ${ }^{3}$ Head of Dept of Surgery, Sudha Hospital \& Medical Research Centre Kota, Rajasthan, India \\ ${ }^{4}$ Department of Obs \& Gynae, RNT Medical College, Udaipur \\ *Corresponding author: jayantsun@yahoo.co.in
}

Received November 06, 2013; Revised December 15, 2013; Accepted December 22, 2013

\begin{abstract}
Extraosseous Ewing's sarcoma/primitive neuroectodermal tumor (ES/PNET) is an uncommon, aggressive, and malignant tumor of poor prognosis. Pancreas in one of the very rare extraosseous location for this tumor thus very minimal information regarding this disorder is present in literature. The present case was clinically and radiologically misdiagnosed as a pancreatic tumor. Histopathology of the tumor tissue revealed "small round cells" that were positive for CD99 (MIC-2), confirming the diagnosis of ES/PNET. In young adults with intraabdominal, extraintestinal mass, Ewing's sarcoma must be kept as a differential diagnosis is our aim in presenting this case report.
\end{abstract}

Keywords: extraosseous ewing's sarcoma, pancreatic carcinoma, atypical pancreatic carcinoma, primitive neuroectodermal tumor

Cite This Article: Kumar Jayant, Swati Agrawal, Rajendra Agarwal, and Susheela Khoiwal, "Pancreatic Ewings Sarcoma- A Dreadful Tumor.” American Journal of Cancer Prevention 1, no. 3 (2013): 24-26. doi: 10.12691/ajcp-1-3-2.

\section{Introduction}

James Ewing in 1921 was first to document this malignant osteolytic tumor, which is mainly formed of small round cells. It was Tefft in 1969 who first described its extraosseous form which is more rarer and resembles intraosseous ES. [1] The main pathogenic factor involved in causation of ES is EWSR1 gene (located at 22q12) translocation $(11 ; 22)$ resulting in EWSR1-FLI1 fusion product.

The (ES/PNET) also shares certain clinical characteristics, such as mostly seen in teenage years, a tendency to rapidly progression, and responsiveness to the same chemotherapeutic regimens and radiation therapy. As occult metastasis is always present hence, chemotherapy is routinely used. Improvements in multimodality treatment have showed better results. EES/PNET has been reported in a variety of sites, including the pancreas, though this is extremely rare. Here we are portraying a case of pancreatic EES/PNET in a 20 year old woman, which is very unusual location of this disorder and very little documentation exists in literature regarding it.

\section{Case Report}

A 20 year old lady presented with complaint of pain upper abdomen on left side since 6 months. There was no history of jaundice, vomiting, diarrhea, or weight loss. On examination there was a left hypogastric mass measuring
$8 \times 8 \mathrm{~cm}$, firm in consistency and moving with respiration, but not falling forward on knee elbow position. No organomegaly was present. Hemoglobin was $12 \mathrm{~g} / \mathrm{dL}$, TLC- 8000/ul, with normal liver and renal functions, albumin-3.5g/dl, CA19.9- 5U/ml. Chest X-ray was normal.

Computed tomography (CT) scan revealed a large, well-defined, heterogenously enhancing mass measuring 8 $\times 10 \mathrm{~cm}$ in the region of body and tail of pancreas. Few hypodense non-enhancing areas suggestive of necrosis or cystic change were observed. Provisional diagnosis of cystic neoplasm of pancreas was made and surgery was planned (Figure 1).

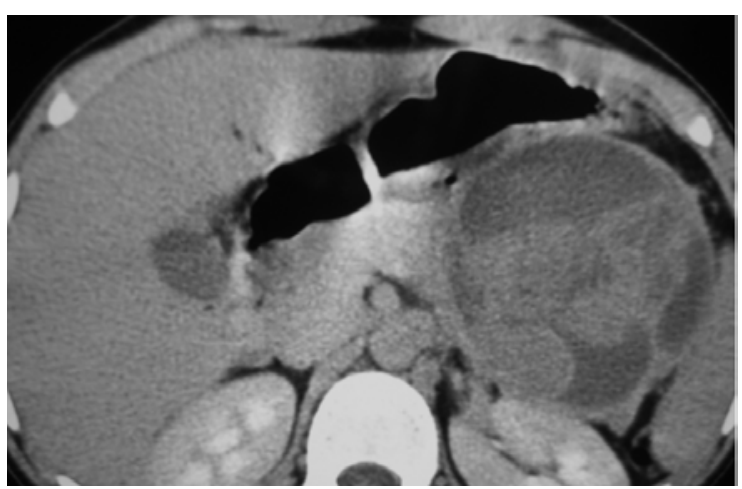

Figure 1. Well-defined, heterogenously enhancing mass in the region of body and tail of pancreas

On exploratory laparotomy, there was tumor arising from body and tail of pancreas which was abutting the left dome of the diaphragm dorsally, the splenic hilum to the left, the transverse mesocolon inferiorly, and the posterior wall of stomach anteriorly. There was no evidence of 
metastasis. (Figure 2) Excision of the tumor with distal pancreatectomy and splenectomy was performed and specimen was sent for histopathological examination. Grossly, the tumor was well circumscribed, capsulated, measured $11 \times 9 \mathrm{~cm}$, and weighed $930 \mathrm{~g}$. The tail of the pancreas was compressed by the tumor and was identified near the splenic hilum. (Figure 3) Cut section revealed a grey tan hemorrhagic solid and cystic tumor with areas of necrosis. (Figure 4).

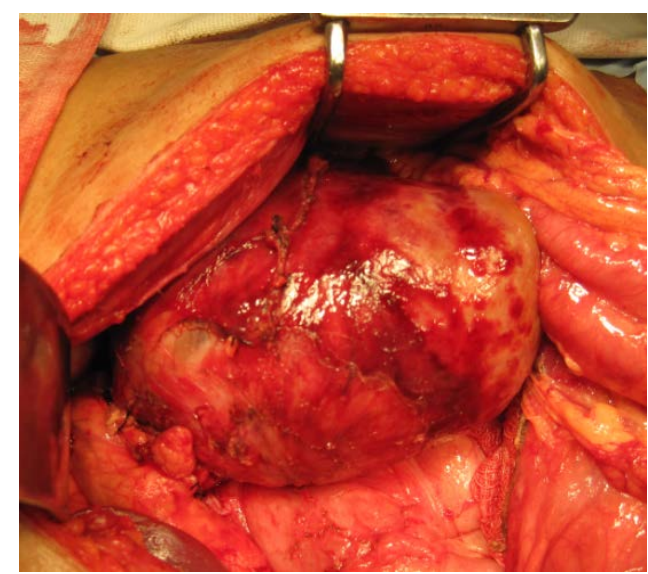

Figure 2. Intraoperative picture showing tumor mass

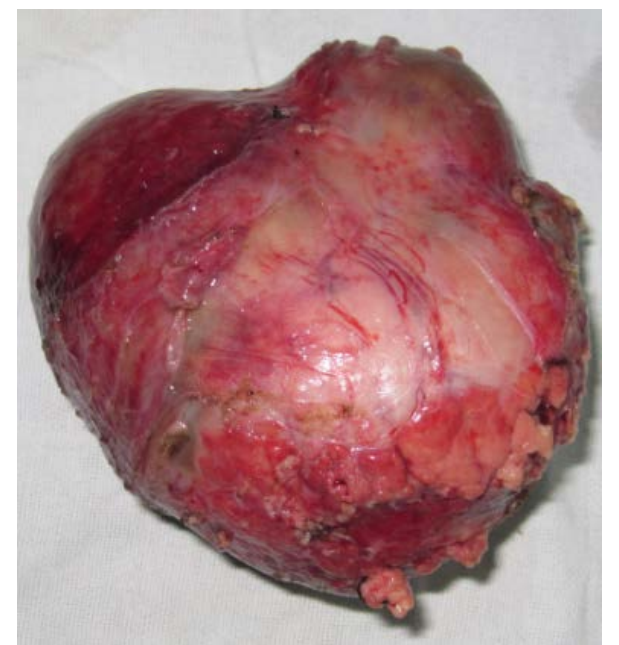

Figure 3. Well-circumscribed and capsulated tumor mass

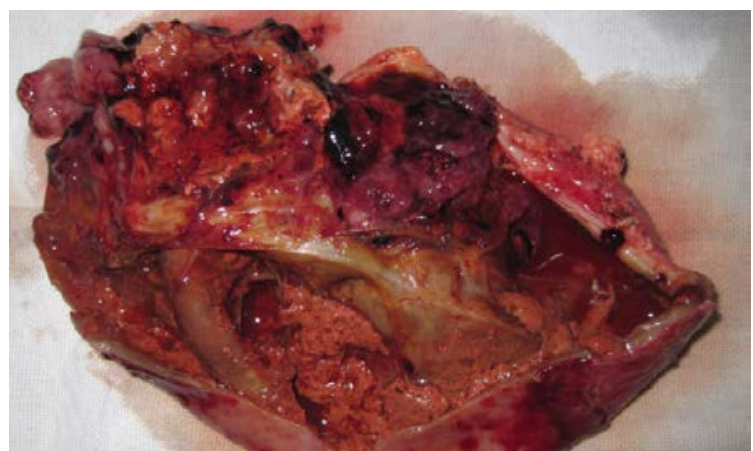

Figure 4. Cut section showed grey tan hemorrhagic solid and cystic tumor with areas of necrosis

On microscopy it was well-circumscribed tumor with a fibrous pseudocapsule composed of sheets of small round cells with enlarged nuclei, fine stippled chromatin, and moderately clear to amphophilic cytoplasm staining periodic acid Schiff stain positive. Adjacent pancreatic tissue was normal with no tumor infiltration (Figure 5, Figure 6). The tumor cells were staining positive for CD
99, while negative for cytokeratin (CK), insulin, glucagon, synaptophysin (SYP), and chromogranin (CHR). (Figure 7, Figure 8) Based on morphology and immunohistochemistry findings, a complete diagnosis of ES/PNET of pancreas was made. Metastatic workup of the patient was negative. Patient recovered well and was discharged on post operative day 6. Follow up was done in out patient department and scheduled chemotherapy was given in form of VAC (vincristine, adriamycin, and cyclophosphamide) along with radiotherapy. She was regularly followed clinically and by ultrasound. After 2 years of surgery she came with complaint of pain abdomen, jaundice and breathlessness. Ultrasound showed mass in hypogastrium, PET scan revealed metastasis in bone, liver and lungs. Palliative chemotherapy and radiotherapy was given but patient deteriorated gradually and her battle of life was finished.

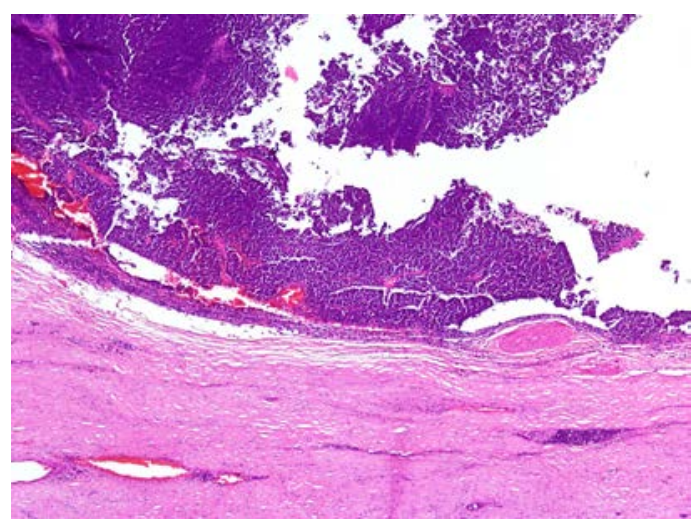

Figure 5. Well-circumscribed tumor with a fibrous pseudocapsule (H\&E stain $\times 400$ magnification)

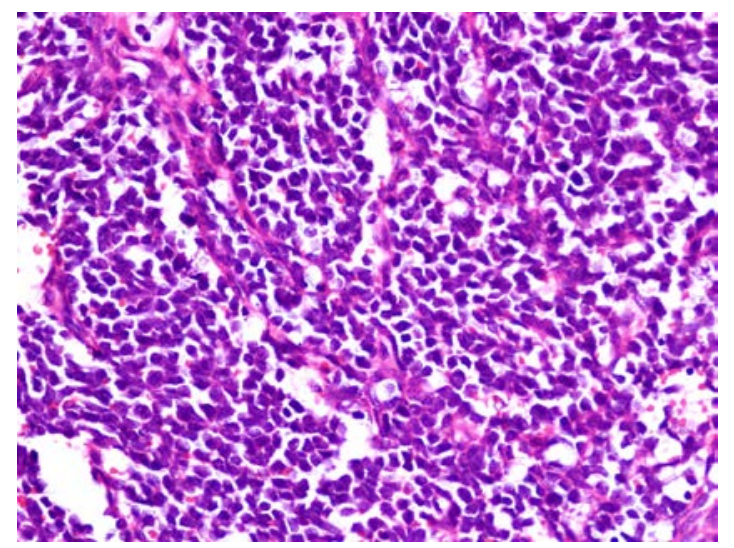

Figure 6. Sheets of small round cells with enlarged nuclei, fine stippled chromatin, and moderately clear to amphophilic cytoplasm (H\&E stain $\mathrm{x}$ 800 magnification)

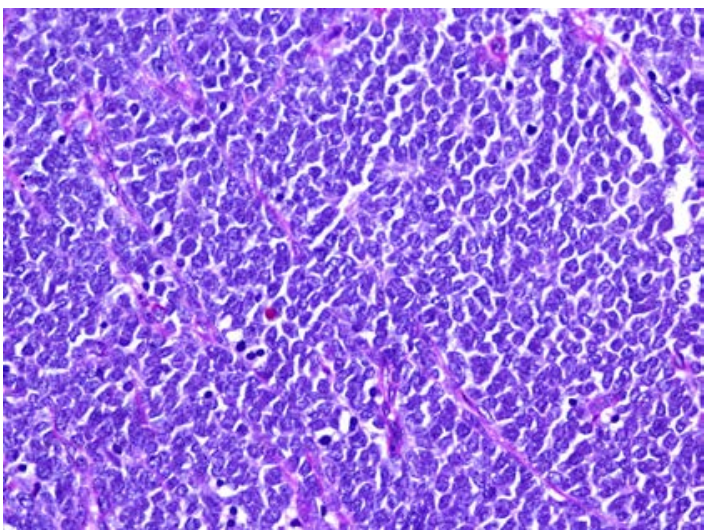

Figure 7. PAS stain positive 


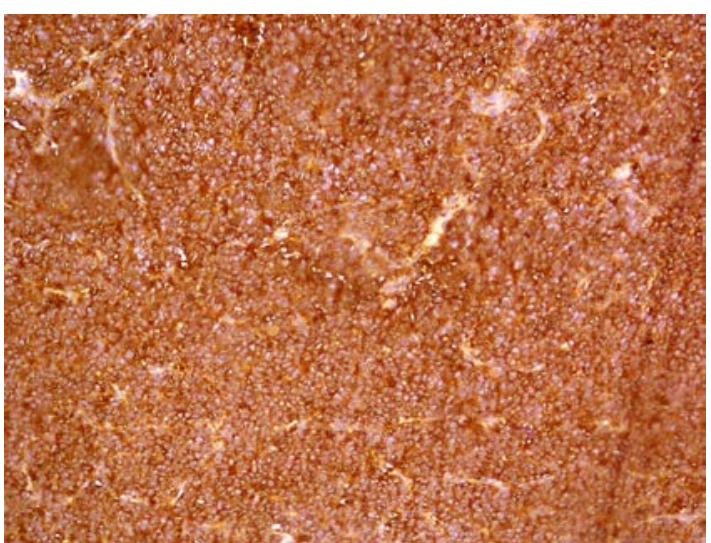

Figure 8. CD-99 positive

\section{Discussion}

ES and PNET are rare tumor arising from ectopic neural and neuroectodermal proliferations, with a clinical possibility to occur anywhere in the body. Because of similar cytogenetic alterations $(\mathrm{t}(11 ; 22)$ which forms EWSR1-FLI1 fusion product), morphologic and immunophenotypic characters they are always documented together as - ES/PNET family of tumors. Common tumors seen in Ewing's sarcoma (ES) family are- classical ES (osseous origin), atypical ES (extraosseous), PNET and Askin tumor. [2] Extraosseous ES/PNET is rare and of real diagnostic challenge because of its poorly differentiated 'small round blue cell' type of tumor histology which is similar to the other tumor seen in retroperitoneum as lymphoma, pancreatic endocrine tumor, pancreatoblastoma, extra-renal Wilm's tumor, neuroblastoma, hepatoblastoma, rhabdomyosarcoma, and visceral small cell neuroendocrine carcinoma [3].

The diagnosis of this tumor requires pre operative work up by USG abdomen and CECT abdomen which per-se are non-specific for diagnosis. Similarly in present case, the tumor was mistaken for a adenocarcinoma type of pancreatic tumor partly because of unanticipated occurrence of this tumor in pancreas. Biopsy is essential for definitive diagnosis, either open biopsy or imaging-guided core biopsy. FNAC is not considered because of smaller tissue sample and lack of tissue architecture. Diagnosis must confirmed by various modalities as immunohistochemistry for CD99 (MIC2 gene) though normal pancreatic tissue and other PNET can express the MIC2 protein, thus limiting specificity of this test. Other modality used is electron microscopic assessment of tumor tissue which shows high nucleus to cytoplasm ratio with glycogen granules in the cytoplasm. [4] Few cells may show neural differentiation with polar processes, which may contain microtubules or neurosecretory glands.

Also molecular genetic studies by polymerase chain reaction (RT-PCR) or FISH detect chromosomal translocation, such as t $(11 ; 22)$ (q24; 12$)$ which is positive in $90-95 \%$ of ES/PNET cases. Diagnosis is finally confirmed with molecular biological examinationwith detection of aberrant chimeric fusion protein, called EWSFLI-1in $90 \%$ of cases [5].

Till now only 14 cases are reported, so exact guidelines are not present to deal with pancreatic ES/PNET. Complete surgical excision is considered to be the main modality of treatment with chemotherapy and radiotherapy as adjuvant with the aim to improve survival and reduce the tumor recurrence. Chemotherapeutic agent tried in combination are vincristine, cyclophosphamide, actinomycin D and doxorubicin. [6] Prognosis is poor and depends on age of patient at the time of diagnosis, tumor bulk, location and prescence secondaries. In our case patient underwent distal pancreatectomy and splenectomy and received adjuvant chemo-radiotherapy, and after 26 months she died of multiple liver, bone and lung metastasis [7].

In conclusion, till date we have hardly 14 cases of PNET of the pancreas exist in literature. This case report is presented to emphasize consideration of ES/PNET in the differential diagnosis of atypical pancreatic tumors in young age group. Also there is more need to understand the biology of this tumor so that better targeted management can be delivered thereby optimizing the potential for curative outcome.

\section{References}

[1] De Alava E, Gerald WL. Molecular biology of the Ewing sarcoma/primitive neuroectodermal tumor family. J ClinOncol 2000; 18:204-213.

[2] Bulchmann G, Schuster T, Haas RJ et al. Primitive neuroectodermal tumor of the pancreas. An extremely rare tumor. Case report and review of the literature. KlinPadiatr 2000; 212: 185-188.

[3] Danner DB, Hruban RH, Pitt HA et al. Primitive neuroectodermal tumor arising in the pancreas. Mod Pathol 1994; 7:200-204.

[4] Perek S, Perek A, Sarman K et al. Primitive neuroectodermal tumor of the pancreas. A case report of an extremely rare tumor. Pancreatology 2003; 3:352-356.

[5] Reilly C, Zenoni S, Hasan MK et al. Primary pancreatic Ewing's sarcoma with portal vein tumor thrombosis. J Gastrointest Surg. 2013 May; 17(5):1015-9.

[6] Moschovi M, Trimis G, Stefanaki K et al. Favorable outcome of Ewing sarcoma family tumors to multiagent intensive preoperative chemotherapy: a single institution experience. J SurgOncol 2005; 89: 239-243.

[7] Eralp Y, Bavbek S, Basaran M et al. Prognostic factors and survival in late adolescent and adult patients with small round cell tumors. Am J ClinOncol 2002; 25: 418-424. 